\title{
147. Incarceration endokardialer Schrittmacherelektroden
}

\author{
G. Fraedrich, J. Mulch, V. Mitrovic und H. H. Scheld \\ Klinik für Herz- und Gefäßchirurgie, Justus-Liebig-Universität, Klinikstraße 25, D-6300 Gießen

\section{Entrapment of Pacemaker Electrodes}

Summary. Infection of pacemaker systems necessitates their entire replacement. Leaving parts of electrodes in situ leads to several major complications such as thrombosis of the great veins or migration of the electrodes. In order to avoid those problems continuous traction should be tried. A total of 13 patients with persistent septicemia, severe rhythm disturbances, or imminent embolism due to retained electrodes had to treated. In 8 of them removal was possible by atriotomy, whereas in $5 \mathrm{ECC}$ has to be instituted to extirpate the electrodes. Key words: Pacemaker electrodes - Entrapment - Continuous traction - Extracorporeal circulation.

Zusammenfassung. Die Infektion eines Schrittmachersystems erfordert seine vollständige Entfernung. Intravasales Belassen des zentralen Anteils der incarcerierten Elektrode führt zu schwerwiegenden Komplikationen wie Thrombose der großen Venen oder Migration des freien Sondenanteils. Zur Vermeidung dieser Komplikationen sollte eine Dauerzugbehandlung versucht werden. Die Zuweisung von 13 Patienten mit gekapptem Sondenanteil und Sepsis oder Lungenembolie erforderte ein aggresiveres Vorgehen. Über eine rechtsseitige Atriotomie in HLM-Bereitschaft gelang bei 8 Patienten die Entfernung der Elektrode während in 5 Fällen die EKK eingesetzt werden mußte.

Schliisselwörter: Schrittmacherelektroden - Incarceration - Dauerzugextraktion - Herz-Lungen-Maschine.

\section{Mezlocillin und Oxacillin als Infektionsprophylaxe in der Herzchirurgie}

\author{
P. Eigel und P. Satter \\ Klinik für Thorax-, Herz- und Gefäßchirurgie, Universitätskliniken Frankfurt/Main, \\ Theodor-Stern-Kai 7, D-6000 Frankfurt 70
}

\section{Mezlocillin and Oxacillin as Prophylaxis Against Infection in Cardiac Surgery}

Summary. Twenty-two patients undergoing cardiac surgery were given $3 \mathrm{~g}$ Optocillin intravenously when anesthesia was introduced. Blood samples and specimens from the right atrium, the resected heart valves and the pectoralis muscle were taken. The concentrations of Oxa/Mezlocillin were measured. At the start of the ECG $159 \mu \mathrm{g} / \mathrm{ml}$ was found in serum samples, $45.5 \mu \mathrm{g} / \mathrm{g}$ in the right atrium and $46.0 \mu \mathrm{g} / \mathrm{g}$ in resected heart valves. The results show Optocillin to be an excellent antibiotic prophylactic agent in cardiac surgery.

Key words: Antibiotic prophylaxis - Cardiac surgery - Mezlocillin - Oxacillin.

Zusammenfassung. Bei 22 konsekutiven am Herzen operierten Patienten wurden $3 \mathrm{~g}$ Optocillin bei Narkoseeinleitung infundiert und in definierten Zeitabständen Serumproben sowie Proben aus dem rechten Herzvorhof, den resezierten Herzklappen und dem Pectoralismuskel entnommen. Die Konzentrationen von Oxa/Mezlocillin wurden bestimmt. Sie lagen bei EKZ-Beginn um $159 \mu \mathrm{g} / \mathrm{ml} \mathrm{im} \mathrm{Serum,} \mathrm{im} \mathrm{Herzohr} \mathrm{betrug} \mathrm{die} \mathrm{Gewebekonzentra-}$ tion $45,5 \mu \mathrm{g} / \mathrm{g}$ und in den Klappen $46,0 \mu \mathrm{g} / \mathrm{g}$. Die gefundenen Serum- und Gewebespiegel weisen die Kombination Oxa/Mezlocillin als geeignetes Infektionsprophylacticum in der Herzchirurgie aus.

Schlïsselwörter: Antibioticaprophylaxe - Herzchirurgie - Mezlocillin - Oxacillin. 